\title{
Chemische Untersuchungen über das Glukomannan aus "Konjak".
}

V. Mitteilung. Methylierung des Glukomannans.

\author{
Von \\ Kitsuji Nishida und Hideo Hashima
}

(Eingegangen am 26 Juni 1931)

Der Methylierungsversuch gibt die Aufklärung über das Konstitutionsproblem des Glukomannans und durch ihn wird ein gewisser eindeutiger Abschluss erreicht. Zunächst wurden $10 \mathrm{~g}$. Glukomannan mit $200 \mathrm{ccm} .15 \%$ iger Natronlauge und $96 \mathrm{~g}$. Dimethylsulfat methyliert. Nach 6-mal Methylierung betrug der Methoxylgehalt $34 \%$. Durch weitere Methylierungen, unter Verwendung von $20 \mathrm{~g}$. Glukomannan, $30 \mathrm{ccm}$. $30 \%$ iger Natronlauge und $288 \mathrm{~g}$. Dimethylsulfat für jeden Methylierungsversuch, war dann der Methoxylgehalt ein höhere geworden und 12 14 malige Methylierung diesen Wert $41.5 \%$ hatte erreichen lassen.

Bei der Methylierung von $2 \mathrm{~g}$. methylierten Glukomannans $(41.6 \% \mathrm{Me}-$ thoxyl) mit $50 \mathrm{ccm}$. Methyljodid und $36 \mathrm{~g}$. Silberoxyd erreichte der Wert nicht über $41.6 \% \mathrm{OCH}_{3}$. Wir müssen hieraus den Schluss ziehen, dass das Glukomannan bei der Methylierung mit Dimethylsulfat und Alkali oder mit Methyljodid und Silberoxyd gleichviel Methoxyl liefert wie die Cellulose und das Lichenin, d. h. dass er nicht über $42 \%$ zunehmen kann, und dass die Methylierung beim Arbeiten mit 30\% iger Natronlauge und bei niedrigerer Temperatur leichter ist.

Das Methylglukomannan $\left(\mathrm{OCH}_{3} 41.6 \%\right)$ ist in kaltem Wasser kolloidal lösbar, in heissem Wasser dagegen tritt wieder Ausflockung ein, wie der Methyläther der Cellulose, des Lichenin und der Stärke. Es löst sich in Chloroform, Bromoform, Alkohol, Aceton, Eisessig und Essigester, doch haben wir eine Molekulargewichtsbestimmung nicht ausgeführt.

Die Hydrolyse des Glukomannanäthers führt zu methyliesten Hexosen, und hat keine Abspaltung von Methylgruppen im Gefolge. Zu diesem Zweck wurden $29.8517 \mathrm{~g}$. methyliertes Glukomannan $\left(\mathrm{OCH}_{3} 41.5 \%\right)$ mit 1\%iger methylalkoholischer Salzsäure in Bonbenröhren zu den methylierten Methylglukosiden und -mannosiden aufgespalten. Das Spaltungsprodukt wurde mit Silberkarbonat neutralisiert und es wurden nach dem Trocknen im Hochvakuum bei der fraktionierten Destillation fünf Fraktionen aufgefangen. Die ersten drei Fraktionen zeigten ein fast farbloses, die späteren ein schwach gelbliches Aussehen, und alle färbten beim Stehen in langer Zeit nach. Die Analyse ergaben. 


\begin{tabular}{rl|c|c|c|c|c|c}
\hline \hline & $\begin{array}{c}\text { Sdp. } \\
\left({ }^{\circ} \mathrm{C}\right)\end{array}$ & \multicolumn{2}{|c|}{$\begin{array}{c}\mathrm{OCH}_{3} \\
\%\end{array}$} & $\mathrm{n}_{\mathrm{D}}^{15}$ & \multicolumn{2}{|c}{$[\alpha]_{\mathrm{D}}^{20}$} \\
\hline I & Fraktion & $124 \sim 125$ & 50.83 & 50.11 & - & - & - \\
II. & Fraktion & $125 \sim 127$ & 51.10 & 50.74 & 1.4604 & $+43.8^{\circ}$ & $+45.6^{\circ}$ \\
III. & Fraktion & $127 \sim 129$ & 48.68 & 48.11 & 1.4587 & $+42.3^{\circ}$ & $+52.8^{\circ}$ \\
IV-A. & Fraktion & $126 \sim 128$ & 47.96 & 48.57 & 1.4597 & $+43.0^{\circ}+42.5^{\circ}$ & - \\
IV-B. & Fraktion & $128 \sim 139$ & 47.16 & 47.08 & 1.4626 & $+45.9^{\circ}$ & $+56.6^{\circ}$ \\
\hline
\end{tabular}

Aus diesen Versuchen geht also hervor, dass die Konstanten der Fraktionen, die aus methyliertem Glukomannan einerseits, aus Methylcellulose und -lichenin anderseits durch Spaltung gewonnen worden sind, eine voreinander erheblich abweichende Zusammensetzung $\mathrm{zu}$ haben scheinen, da das Glukomannan aus $2 \mathrm{Mol}$ Mannose und $1 \mathrm{Mol}$ Glukose besteht und die Spaltprodukte müssen Trimethylmannosid und -glukosid enthalten.

Die I. und II. Fraktion stehen im Methoxylgehalt ca. 1.1\% unter denjenigen, welche sich für ein Trimethylmethylmannosid oder -glukosid aussprechen lassen wurden $(52.6 \%)$; es muss darin kleine Mengen Dimethylmethylmannosid oder -glukosid $(41.8 \%)$, das in der III.-IV. Fraktion überwiegt, enthalten sein. Der Methoxylgehalt der II. Fraktion war sehr gleichmässig, aber Brechungsindex war etwas hoch, und die spezifische Drehung war klein, verglichen nicht mit denjenigen, die bei Methylcellulose und -lichenin durch Abbau erhalten werden.

Die II. Fraktion wurde mit wässeriger Salzsäure verseift und dabei schied sich nicht die kristallisierte 2,3,6-Trimethylglukose $\left(41.89 \% \mathrm{OCH}_{3}\right)$ nach langem Stehen wie diejenige, die aus der ersten Fraktion von Methylbaumwolle und Methylolichenin abgebaut wurde, ab. Dieser Sirup erwies:

$$
\% \mathrm{OCH}_{3} \quad 40.49 ; 40.49 \text {. }
$$

Es muss darin eine Spur Dimethylhexose $\left(29.81 \% \mathrm{OCH}_{3}\right)$ enthalten sein. Wir haben diesen Sirup mit Salpetersäure (spez. Gew. 1.20) oxydiert und erhielten Trimethylzuckersäurelakton oder -mannozuckersäureiakton mit folgenden Eigenschaften :
$\mathrm{OCH}_{3}$-Gehalt gef.
Berechnet für Trimethylzuckersäurelakton
$37.15 ; 36.89 \%$ $39.74 \%$
Verbrauch von $N / 10$-Natronlauge.
$10.00 \mathrm{ccm}$. (Substanz $0.116 \mathrm{~g}$.)

Aus diesem Ergebnis geht also hervor, dass die I. und Il. Fraktion 2, 3, 4-Trimethylhexosid waren mit kleine Mengen Dimethylhexosid und nicht 2, 3, 6-Trimethylmethylhexosid, und mit grosser Wahrschcinlichkeit handelte es sich um 2, 3, 4-Trimethylglukosid und nicht Mannosid. Die III, IV-A und -B Fraktion wurden auch wie die erste Fraktion mit Salzsäure verseift, dabei wir Trimethylhexose erhielten, die nach langem Stehen keine Kristalle 
abschied. Die Analyse ergab :

\begin{tabular}{c|cc|c}
\hline Fraktion & \multicolumn{2}{|c|}{$\% \mathrm{OCH}_{3}$} & {$[\alpha]_{\mathrm{D}}^{20}$} \\
\hline III. & 38.04 & 38.01 & $+36^{\circ}$ in Wasser \\
IV-A & 38.73 & 38.46 & $+18.5^{\circ}$ in Chloroform \\
IV-B & 36.26 & 36.38 & $+11.6^{\circ}$ in Chloroform \\
\hline
\end{tabular}

Im Methoxylgehalt stehen diese Fraktionen ca. 3.3 5.6\% unter der Trimethylhexose; es muss darin bereits geringe Mengen Dimethylhexose enthalten sein. Dies Fraktion wurden wie oben mit Salpetersäure oxydiert, und dabei wurden Mono- und Dikarbonsäurelakton mit folgenden Eigenschaften gewonnen.

Aus der III. Fraktion

Aus der IV $-\Lambda$ Fraktion

$\mathrm{OClI}_{3}$-Gehalt gef. $36.12 ; 35.78$

Aus der IV-B Fraktion

$\mathrm{OCH}_{3}$-Gehalt gef. $33.89 ; 33.76$

\begin{tabular}{|c|c|c|c|c|c|c|c|}
\hline \multicolumn{2}{|c|}{ Fraktion } & \multirow{2}{*}{$\begin{array}{l}\text { Substanz } \\
0.1071 \mathrm{~g} .\end{array}$} & \multirow{2}{*}{$\begin{array}{c}\text { Verbrauch } \\
\text { von } N / 10- \\
\text { Natronlauge } \\
7.52 \mathrm{ccm}\end{array}$} & \multicolumn{2}{|c|}{ Fraktion } & \multirow{2}{*}{$\begin{array}{c}\text { Substanz. } \\
0.1180\end{array}$} & \multirow{2}{*}{ 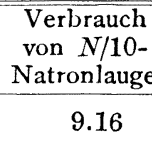 } \\
\hline III. & Fraktion & & & IV $-B$ & Fraktion & & \\
\hline IV $-A$ & Fraktion & 0.1762 & 9.98 & IV-B & Fraktion & 0.2631 & 19.33 \\
\hline
\end{tabular}

Aus hier nahm in der III. und der IV-B Fraktion die Acidität $z u$, während der Säuregehalt in der IV-A Fraktion zur Hälfte zurückging. Bei all diesem Säurelakton handelte es sich selbstverständlich um Mischungen, aus denen die erste (aus der III Fraktion) eine Lösung von Trimethylzuckersäurelakton mit kleinen Mengen Trimethylmannonsäurelakton, die zweite (aus der IV-A Fraktion) ein Sirup von Trimethylmannonsäurelakton mit einer Spur Tri- und Dimethylmannozuckersürelakton, die dritte (aus der IV-B Fraktion) eine Mischung von Tri- und Dimethylmannozuckersäurelakton waren. Bei 2, 3, 6-Trimethylmethylglukosid Fraktion finden sich nach Verseifung mit Salzsäure bekanntlich Kristalle von 2, 3, 6-Trimethylglukose, die mit Salpetersäure zu 2, 3,6-Trimethylglukonsäurelakton (Monokarbonsäurelakton) oxydieren müssen. Der grössere Teil aus der IV-A Fraktion war 2, 3, 6Trimethylmethylhexosid, aber seine Eigenschaften und das Ergebnis ihrer Analyse zeigte nicht so grosse Aehnlichkeit, dass es sich hier um 2, 3, 6Trimethylmethylglukosid handeln konnte; der Versuch, in welchem aus methyliertem Glukomannan durch Spaltung keine Kristalle gewonnen wurden, bildete also auch eine Stütze für die Abwesenheit der 2, 3, 6-Trimethylglukose. Da die Trimethylglukose und -mannose mit Phenylhydrazin kein Phenylosazon liefert, so muss am Kohlenstoffatom 2 eine Methoxylgruppe sitzen. Als Gesamtergebnis der im vorstehenden skizzierten Untersuchungen auf dem 
Gebiete des Methylglukomannans folgt, dass das Spaltprodukt auf die Weise gewonnen wurde, wie sie das nachstehende Schema zeigt:

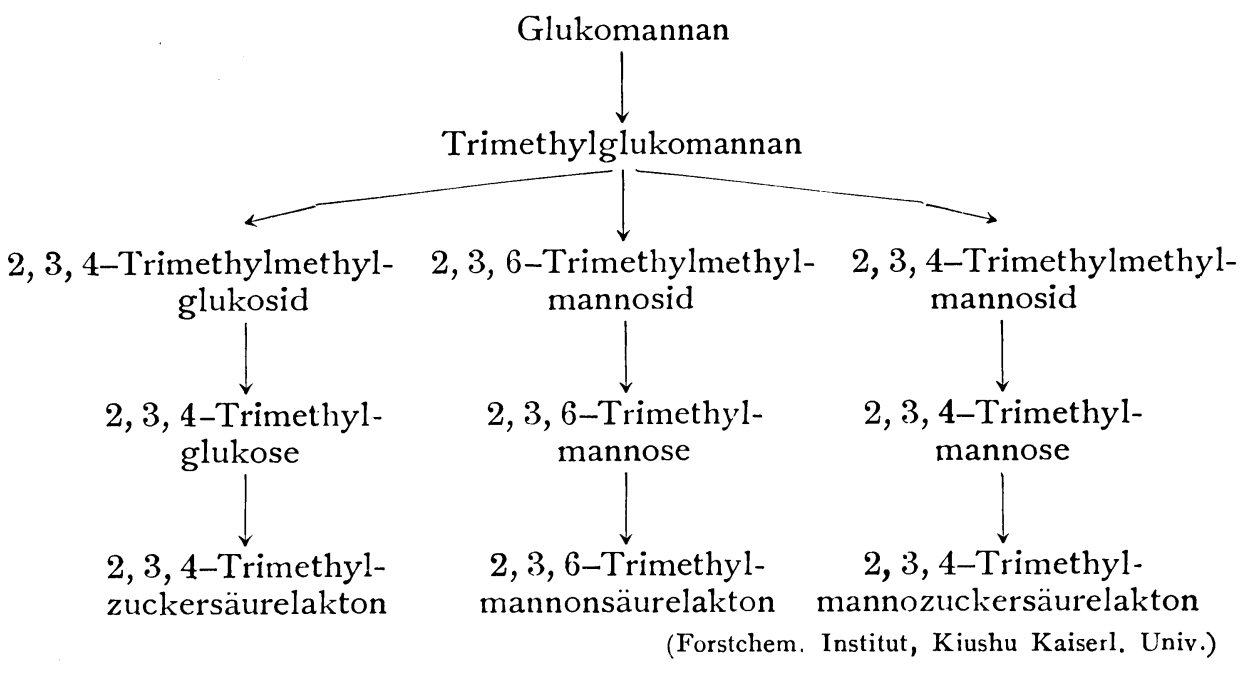

\section{Untersuchung über das ätherische Oel aus Podocarpus macrophylla Don. III.}

Mitteilung. Ueber die oxydierenden Produkten der neuen Diterpene ( $\alpha$ - und $\beta$-Podocarpren).

Von

Kitsuji Nishida und Hidetaka Uota

(Eingegangen am 26 Juni 1931)

Zur Konstitutionsaufklärung des $\alpha$ - und $\beta$-Podocarpren führten wir den oxdierenden Abbau der Diterpene (1) mit Kaliumpermanganatlösung (2) mit Braunstein und Schwefelsäure (3) mit Kaliumbichromat und Schwefelsäure (4) mit Kaliumpermaganatlösung bei Gegenwart von Alkali durch. Es wurde dabei (4) neben Terpen und carboxylierten Produkte, $\beta$-Podocarprenoxycarbonsäure, $\beta$-Podocarprenketocarbonsäure, noch Diketo- $\alpha$-Podocarpren erhalten wie folgende Schema:

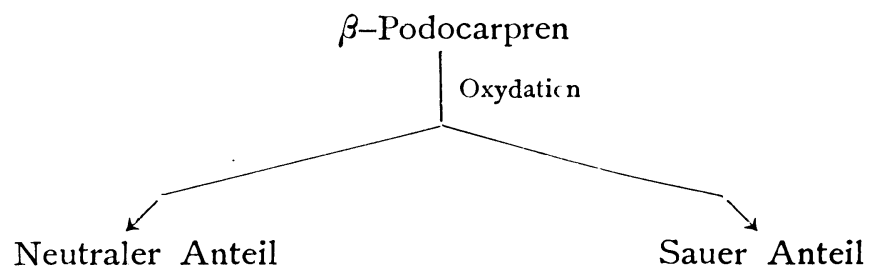

